\title{
Real-time implementation of airborne inertial-SLAM
}

\author{
Jonghyuk Kim ${ }^{\mathrm{a}, *}$, Salah Sukkarieh ${ }^{\mathrm{b}}$ \\ ${ }^{a}$ College of Engineering and Computer Science, The Australian National University, ACT 0200, Australia \\ $\mathrm{b}^{\mathrm{b}}$ ARC Center of Excellence for Autonomous Systems, The University of Sydney, NSW 2006, Australia
}

Received 1 October 2005; received in revised form 1 April 2006; accepted 1 June 2006

Available online 26 September 2006

\begin{abstract}
This paper addresses some challenges to the real-time implementation of Simultaneous Localisation and Mapping (SLAM) on a UAV platform. When compared to the implementation of SLAM in 2D environments, airborne implementation imposes several difficulties in terms of computational complexity and loop closure, with high nonlinearity in both vehicle dynamics and observations. An implementation of airborne SLAM is formulated to relieve this computational complexity in both direct and indirect ways. Our implementation is based on an Extended Kalman Filter (EKF), which fuses data from an Inertial Measurement Unit (IMU) with data from a passive vision system. Real-time results from flight trials are provided.
\end{abstract}

(C) 2006 Elsevier B.V. All rights reserved.

Keywords: Airborne SLAM; Inertial Measurement Unit (IMU); Vision; UAV

\section{Introduction}

Unmanned Aerial Vehicles (UAVs) have attracted much attention from robotics researchers in both civilian and defense industries over the past few years. They can perform various tasks in highly dangerous environments, where access by human operators or from ground vehicles are limited. There is a broad spectrum of applications, ranging across academic research, resource monitoring, search and rescue, bush fire monitoring and information gathering. Advances in cost effective inertial sensors and accurate navigation aids, such as the Global Navigation Satellite System (GNSS), have been key determinants of the feasibility of UAV systems. By fusing information from an Inertial Measurement Unit (IMU) with that from GNSS, a 6 DoF vehicle state can be reconstructed, which is a crucial step for guidance and flight control [1,2].

In many robotics applications however, the vehicle needs to perform a task within environments where GNSS information may not be available, such as indoors, in forests, underground, or other such locations where GNSS is naturally denied. In such cases, autonomous localisation is required.

\footnotetext{
* Corresponding author.

E-mail address: jonghyuk.kim@anu.edu.au (J. Kim).
}

Autonomous localisation is a process of determining the platform's position without the use of any a priori information external to the platform except for what the platform senses about its environment; that is, the determination of the platform's position and attitude without the use of predefined maps or any purpose-built infrastructure. This is also known as Simultaneous Localisation and Mapping (SLAM), as introduced by [3], where the vehicle has a capability for online map building, while simultaneously utilising the generated map to estimate and correct errors in the navigation solution obtained.

There have been significant advances in SLAM research over recent years in terms of its real-time deployment and implementation on land, and in underwater applications. Most efforts have concentrated around reducing the computational complexity of SLAM. For example, large-scale maps are partitioned into small amenable maps [4,5] and [6] introduced the hierarchical sub-map method. The sparse nature of the SLAM information filter has also been extensively investigated and implemented [7]. In parallel to these efforts, there have been attempts to develop SLAM for 3D environments, for example: the use of rotating laser range finders in mining applications [8], and the use of stereo vision systems for lowdynamic aerial vehicles [9]. However, in these applications, the 3D implementation is limited to the use of low-dynamic 


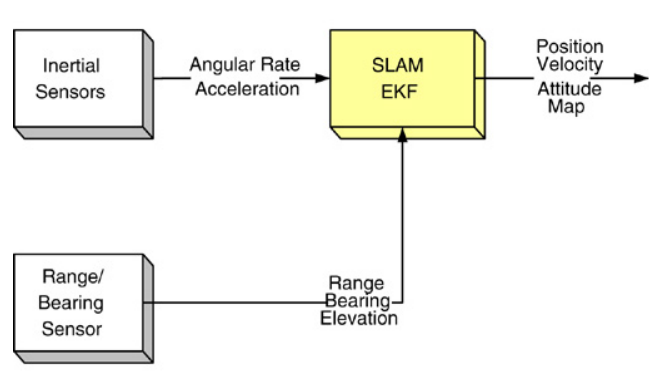

(a)

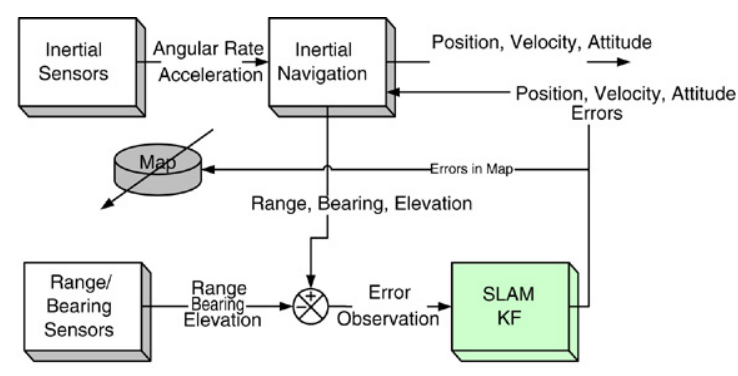

(b)

Fig. 1. (a) The direct 6DoF SLAM structure, which estimates the vehicle position, velocity and attitude along with observed feature locations, and (b) the indirect 6DoF SLAM structure, which uses the error state of the INS and map model, and estimates the errors in the vehicle states and map.

vehicles, due to the extensive processing needed for $3 \mathrm{D}$ mapping.

For airborne applications, to the best of our knowledge there have been only three attempts up to now: SLAM on a blimp-type (thus low-dynamic) platform using a stereo vision system [9]; inertial SLAM in a laboratory environment [10]; and SLAM on a fixed-wing UAV with inertial sensors and a single vision system by the present authors [11].

In this paper, we will provide a real-time implementation of airborne SLAM as an extension of our previous work. The challenge in airborne deployment of SLAM lies in the complexity of the dead-reckoning process involved and its fast-drifting error. If we look at how the localisation system for an airborne vehicle has been formulated in the past, the core sensing device has been an IMU. This unit measures the acceleration and rotation rate of a platform with high update rates, which can then be transformed and processed to provide its position, velocity and attitude, resulting in an Inertial Navigation System (INS) $[12,13]$. The data presented by the INS are fed to the guidance and control system to achieve further autonomy. Inertial navigation is significant in that it only measures dynamical quantity, and is thus independent of platform kinematics. The navigational solution provided by INS, however, drifts with time, as in most other deadreckoning systems. However, the drift rate of the inertial position is typically a cubic function of time, which makes the development of any inertially based SLAM a challenge. Even small errors in gyros will be accumulated in angle estimates (roll and pitch), which in turn misrepresent gravitational acceleration as the vehicle acceleration, thus resulting in quadratic velocity (and cubic position) errors. Therefore, the INS requires reliable and effective supplementary information to constrain these errors. In this paper, we will provide results from a real-time airborne SLAM based on an Extended Kalman Filter (EKF), which fuses information obtained from a vision system with the information from the INS.

In Section 2, we will present our airborne SLAM algorithm based on two different approaches, direct and indirect, and discuss the benefits of both. Sections 3 and 4 will provide details of the real-time SLAM implementation and flight test results. Section 5 provides a conclusion, with directions for future work.

\section{Airborne SLAM formulation}

SLAM has been formulated directly for nonlinear dynamic and observation models, using an EKF. In inertial navigation applications however, SLAM can also be formulated indirectly for linearised error dynamic/observation models, using a linear Kalman Filter (KF). This indirect formulation has several benefits over the direct formulation. Fig. 1 compares these two SLAM structures. In both cases, an IMU provides the acceleration and angular velocity of the vehicle. The observation sensor provides the range, bearing and elevation of observed features. In direct form, the filter accepts raw data from the IMU and passes this into a nonlinear $6 \mathrm{DoF}$ model, and the EKF proceeds through the process of predicting and updating the states of the vehicle and feature locations.

In an indirect implementation however, the inertial loop is separated from the filter; thus, the inertial navigation equations transform the raw inertial data to position, velocity and attitude measurements outside of the filter with sufficiently high rates. The state dynamic model in KF is an error model of both the vehicle and the observed features. When an observation occurs, a predicted observation is also generated, which is based on the current location of the vehicle and location of the feature as indicated by the map. The difference between the predicted and actual observations is passed to the $\mathrm{KF}$ as an observed error. The KF uses this to estimate the inertial and feature errors. The estimated errors from the filter are then fed back to the INS and map to make further corrections.

Although the heart of the SLAM algorithm is exactly the same, the main benefits of this indirect structure can be summarised as follows [14]:

- The system becomes more tractable for real-time processing. The main INS loop can provide continuous navigation data within fixed time intervals. The SLAM update cycle, whose computation time increases with map size, will not disrupt the main INS loop, and time propagation algorithms can be used to match information at appropriate times.

- The SLAM prediction cycle can exploit the low-dynamic characteristics of INS errors. As a result, the rate of the prediction cycle can be much lower than that of the direct filter. The more accurate the IMU, the less frequently the prediction cycle has to run. 
- The observability of the system can be analytically investigated using the time-variant linear SLAM model. The observability, Grammian matrix and the rank of the system can thus be evaluated.

The most significant benefit of indirect SLAM is thus its real time performance, if this is required. At the time of publication, only the direct method had been implemented, as it worked sufficiently for our purposes; the indirect form had not yet been implemented because of flight trial constraints. Both SLAM models are presented in the following sections.

\subsection{Direct formulation}

The direct SLAM filter consists of a 6DoF INS and a map model, together with a nonlinear feature observation model. By assuming a local tangent inertial frame, the SLAM equations in continuous time can be written as,

$$
\left[\begin{array}{c}
\dot{\mathbf{p}}^{n} \\
\dot{\mathbf{i}}^{n} \\
\dot{\mathbf{q}}^{n} \\
\hline \dot{\mathbf{m}}_{1 \ldots N}^{n}
\end{array}\right]=\left[\begin{array}{c}
\mathbf{v}^{n} \\
\mathbf{C}_{b}^{n} \mathbf{f}^{b}+\mathbf{g}^{n} \\
\frac{1}{2} \mathbf{q}^{n} \otimes \overline{\boldsymbol{\omega}}^{b} \\
\hline \mathbf{0}_{1 \ldots N}
\end{array}\right],
$$

where $\mathbf{C}_{b}^{n}$ is a directional cosine matrix (DCM) that transforms the specific force vector measured in the body frame to the navigation frame, $\otimes$ represents the quaternion product, and $\overline{\boldsymbol{\omega}}^{b}$ is the quaternion form of the angular velocity measured from the gyros, and $N$ is the total number of features observed.

The onboard terrain sensor measures range, bearing and elevation, and thus provides the relative position vector between the vehicle and observed feature. The relationship between the observation in the sensor frame and the location of the feature in the navigation frame determines the derivation of the observation model. The $i$ th feature position, $\mathbf{m}_{i}^{n}$ in the navigation frame, is a function of the vehicle position $\mathbf{p}^{n}$, the sensor lever-arm offset from the body centre $\mathbf{r}_{b s}^{b}$, and the relative position of the feature, as measured from the sensor location $\mathbf{r}_{s m}^{s}$ in the sensor frame [11]:

$\mathbf{m}_{i}^{n}=\mathbf{p}^{n}+\mathbf{C}_{b}^{n} \mathbf{r}_{b s}^{b}+\mathbf{C}_{b}^{n} \mathbf{C}_{s}^{b} \mathbf{r}_{s m}^{s}$.

Here $\mathbf{C}_{s}^{b}$ is a DCM which transforms the vector in the sensor frame to the body frame, and is defined for each payload sensor installment. A vision camera, installed pointing downward, is used; hence its frame is defined by rotating the body axes $-90^{\circ}$ along the pitch axis. The relative position vector of the map

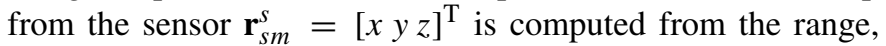
bearing and elevation measurements using a polar-to-Cartesian transformation. The observation model is then obtained by rearranging Eq. (2) and by representing the range, bearing and elevation with respect to the vehicle state and map.

\subsection{Indirect formulation}

As mentioned previously, the indirect SLAM filter estimates the errors in the INS and the map. The error model can be developed by perturbing the nonlinear equation, and can be written as:

$$
\left[\begin{array}{c}
\delta \dot{\mathbf{p}}^{n} \\
\delta \dot{\mathbf{v}}^{n} \\
\delta \dot{\psi}^{n} \\
\hline \delta \dot{\mathbf{m}}_{1 \cdots N}^{n}
\end{array}\right]=\left[\begin{array}{c}
\boldsymbol{\delta} \mathbf{v}^{n} \\
\mathbf{f}^{n} \times \boldsymbol{\delta} \boldsymbol{\psi}^{n}+\mathbf{C}_{b}^{n} \boldsymbol{\delta} \mathbf{f}^{b} \\
-\mathbf{C}_{b}^{n} \boldsymbol{\delta} \boldsymbol{\omega}^{b} \\
\hline \mathbf{0}_{1 \cdots N}
\end{array}\right],
$$

with $\delta \boldsymbol{\psi}^{n}$ being the misalignment angle, $\delta \mathbf{f}^{b}$ being the acceleration error in the body frame and $\delta \boldsymbol{\omega}^{b}$ being the gyro error in the body frame.

To obtain a linearised observation model, the error in an initialised feature position needs to be analysed first. The feature error is defined as the difference between the predicted position $\hat{\mathbf{m}}_{i}^{n}$ and true position $\mathbf{m}_{i}^{n}$ :

$\delta \mathbf{m}_{i}^{n} \triangleq \hat{\mathbf{m}}_{i}^{n}-\mathbf{m}_{i}^{n}$.

Perturbing Eq. (2) with the errors, and subtracting it from the true feature position gives

$$
\begin{aligned}
\delta \mathbf{m}_{i}^{n}= & \left(\hat{\mathbf{p}}^{n}-\mathbf{p}^{n}\right) \\
& +\left(\hat{\mathbf{C}}_{b}^{n} \hat{\mathbf{r}}_{b s}^{b}-\mathbf{C}_{b}^{n} \mathbf{r}_{b s}^{b}\right)+\left(\hat{\mathbf{C}}_{b}^{n} \hat{\mathbf{C}}_{s}^{b} \hat{\mathbf{r}}_{s m}^{s}-\mathbf{C}_{b}^{n} \mathbf{C}_{s}^{b} \mathbf{r}_{s m}^{s}\right) .
\end{aligned}
$$

Using the predicted DCM as the true DCM misaligned by errors, we get:

$\hat{\mathbf{C}}_{b}^{n} \triangleq\left(\mathbf{I}-\left[\delta \psi^{n} \times\right]\right) \mathbf{C}_{b}^{n}, \quad \hat{\mathbf{C}}_{s}^{b} \triangleq\left(\mathbf{I}-\left[\delta \boldsymbol{\theta}_{s}^{b} \times\right]\right) \mathbf{C}_{s}^{b}$,

where $\left[\boldsymbol{\delta} \boldsymbol{\psi}^{n} \times\right]$ and $\left[\boldsymbol{\delta} \boldsymbol{\theta}_{s}^{b} \times\right]$ are the INS attitude error and sensor misalignment error expressed in a skew-symmetric form, respectively. Using these, Eq. (5) can now be rewritten as,

$$
\begin{aligned}
\delta \mathbf{m}_{i}^{n}= & \boldsymbol{\delta} \mathbf{p}^{n}+\mathbf{C}_{b}^{n} \boldsymbol{\delta} \mathbf{r}_{b s}^{b}+\mathbf{C}_{b}^{n}\left[\mathbf{r}_{b s}^{b} \times\right] \boldsymbol{\delta} \boldsymbol{\psi}^{n}+\mathbf{C}_{b}^{n} \mathbf{C}_{s}^{b} \boldsymbol{\delta} \mathbf{r}_{s m}^{s} \\
& +\mathbf{C}_{b}^{n} \mathbf{C}_{s}^{b}\left[\mathbf{r}_{s m}^{s} \times\right]\left(\boldsymbol{\delta} \boldsymbol{\psi}^{n}+\boldsymbol{\delta} \boldsymbol{\theta}_{s}^{b}\right) .
\end{aligned}
$$

The sensor lever-arm error and misalignment error can be calibrated precisely; hence by ignoring them and using a transformed notation for the relative range vector, Eq. (7) can be simplified as,

$\delta \mathbf{m}_{i}^{n}=\delta \mathbf{p}^{n}+\left[\mathbf{r}_{s m}^{n} \times\right] \boldsymbol{\delta} \boldsymbol{\psi}^{n}+\delta \mathbf{r}_{s m}^{n}$.

From this analysis, it is obvious that the position error of the vehicle is directly reflected onto the initialised feature position error, which establishes the correlation structure between the vehicle and observed features. The actual observation input to the filter is the last error term in Eq. (8). Hence rearranging this equation gives the observation model:

$\delta \mathbf{z}_{i}^{n}=\delta \mathbf{m}_{i}^{n}-\delta \mathbf{p}^{n}-\left[\mathbf{r}_{s m}^{n} \times\right] \boldsymbol{\delta} \boldsymbol{\psi}^{n}$.

\subsection{Prediction and update}

With the state transition and observation models defined, the prediction and estimation procedure can proceed. If the direct filter is used, then the nonlinear state model is used to propagate the vehicle state, and the nonlinear observation model is used to estimate the vehicle and map state from 


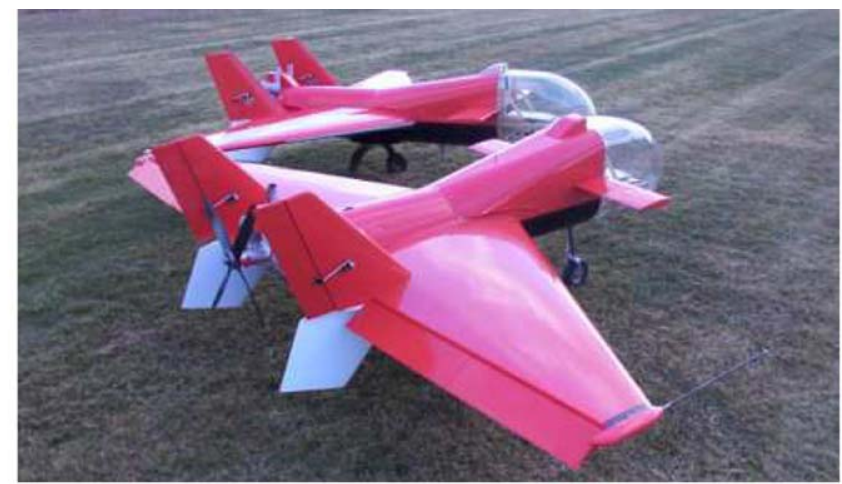

(a)

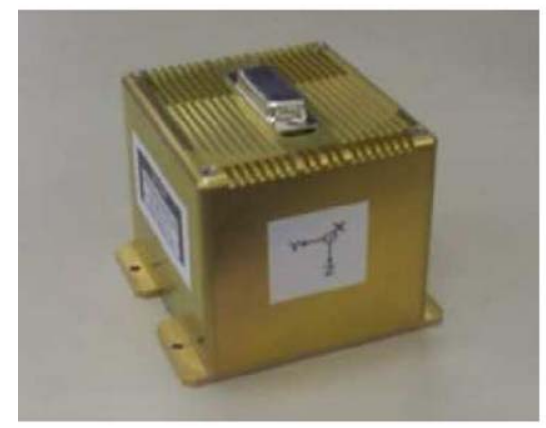

(b)

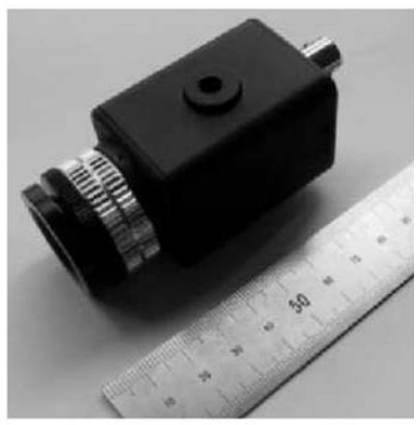

(c)

Fig. 2. (a) Two Brumby MkIII airframes on the runway at the flight test facility, (b) The IMU from Inertial Science that is used to provide 6 DoF vehicle information, and (c) The small Sony camera that is used as the vision system.

the feature observations. The error covariance is propagated and updated by evaluating the Jacobian matrix for nonlinear models. If the indirect filter is used, then the filter predicts only the error covariance, since the predicted errors become zero with the assumption of zero mean Gaussian noise. If feature observations are available, then the error states are estimated in addition to the error covariance matrix. The estimated errors are then applied to the external INS loop and map database for making corrections.

Whenever a feature is observed, data association is conducted, which checks to see if the feature has been previously observed. If the feature has been previously registered on the map, the observation is used to update its state and covariance; if the feature is a new one, then the filter state is augmented by a new feature error state. The data association can be performed by using a simple nearest neighbour method, or by using more robust methods such as joint compatibility/ bound and branch. In this work, the nearest neighbour method is used for its simplicity and ease for real-time implementation. To avoid any possible ambiguity during flight, the ground features are installed using sufficient separation distances.

This indirect inertial SLAM was implemented and compared to the direct form using a high fidelity UAV simulator [14], showing the former's improved performance in terms of the computational complexity and flexibility in filter configuration. In real-time operations, however, only the direct filter was implemented and verified as mentioned, due to the limited schedule of flight trials available, and its results are presented in Section 4.

\section{Real-time implementation}

The (direct) airborne SLAM algorithm was implemented on a UAV platform, namely the Brumby MkIII, which was designed and built at the University of Sydney.

\subsection{Flight vehicle and sensor systems}

The Brumby airframes shown in Fig. 2(a) are capable of flying at approximately $50 \mathrm{~m} / \mathrm{s}$ and have an endurance of the order of 45 min with a $20 \mathrm{~kg}$ payload. Each UAV platform is equipped with various kinds of sensors. All sensors are connected to the Flight Control Computer (FCC) via a serial link or local bus system. An IMU from Inertial Science is used (shown in Fig. 2(b)). It is small in size and lightweight, with a low power consumption, making it ideal for UAV applications. It is classified as a low-grade tactical IMU. It outputs acceleration and rotation rate data at the rate of $400 \mathrm{~Hz}$ to the FCC and SLAM computer through an RS-422 serial link during flight operations. An onboard vision system provides feature observations to the onboard SLAM node. The vision system makes use of a low-cost monochrome camera from Sony, as shown in Fig. 2(c). This is a charge-coupled device which has a resolution of 600 horizontal lines. It has a composite video output, which gives images at $25 \mathrm{~Hz}$ or $50 \mathrm{~Hz}$, depending on the interlaced mode. The vision sensor is mounted pointing down, in the second payload bay of the Brumby MkIII, immediately behind the forward bulkhead. Artificial features were placed on the ground; white plastic 


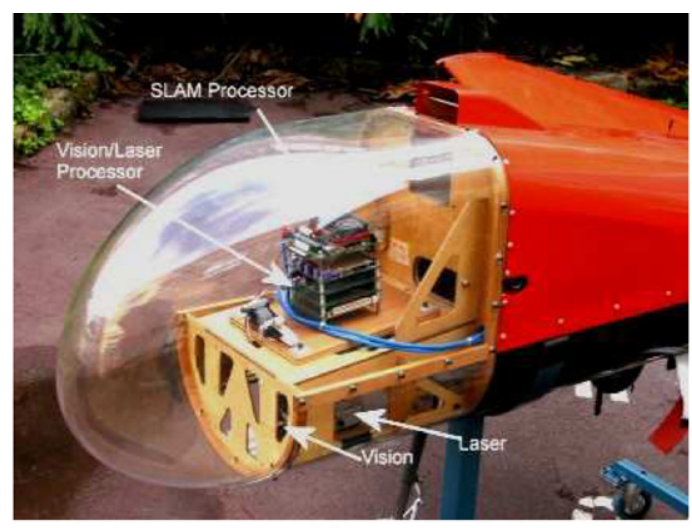

(a)

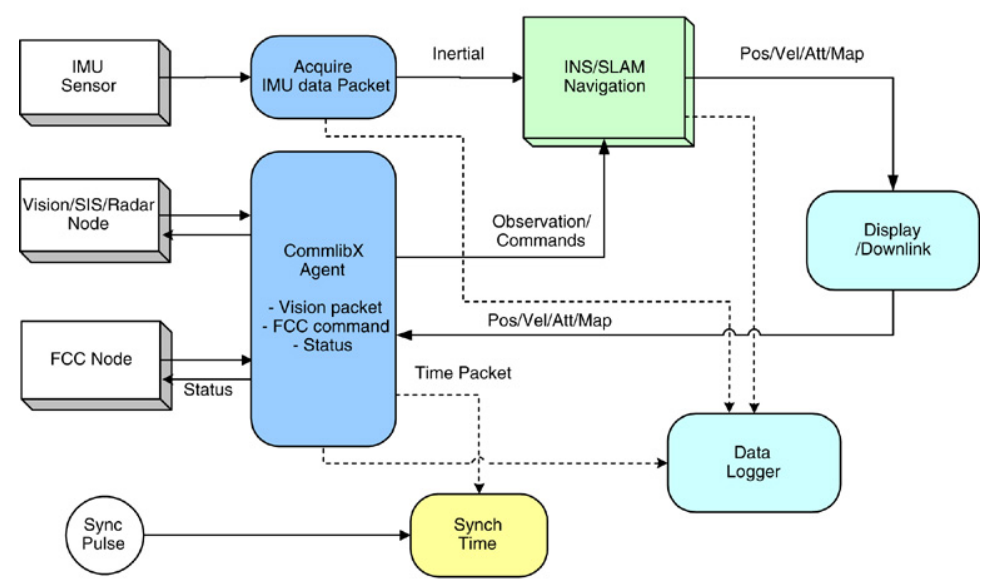

(b)

Fig. 3. (a) The SLAM processor is installed with the front nose pointing downward, and (b) the real-time SLAM multi-threaded structure.

sheets, which were easier for the vision system to identify, were used. A simple intensity threshold algorithm is used to extract these features from the images in real-time. The bearing to each feature can then be generated. Although the vision sensor does not give ranges directly, an estimated value is generated based on the size of the features observed. The SLAM processing computer is installed separately inside the front nose and is shown in Fig. 3(a). The FCC and SLAM computer are connected via the local bus, and all the information required for SLAM operation is passed on through this bus.

\subsection{Real-time software development}

The SLAM algorithm is implemented using the $\mathrm{C}++$ class Framework, which provides flexibility and modularity. By using Windows ${ }^{\mathrm{TM}}$ development tools, the algorithm can be easily debugged and verified, reducing the overall development time needed. Once this has been done, the $\mathrm{C}++$ class modules developed are ported as a multi-threaded real-time SLAM software program under the $\mathrm{QNX}^{\mathrm{TM}}$ environment. The thread inter-connections and data flow within the SLAM computer are shown in Fig. 3(b). As the direct SLAM algorithm is implemented, the filter thread processes the IMU data to predict the vehicle and map states, and to propagate the vehicle covariance and cross-correlation between vehicle and map. Whenever an observation arrives, the filter thread performs a
Table 1

EKF-SLAM filter Q/R Tuning Parameters $(1 \sigma)$

\begin{tabular}{llll}
\hline Param & Type & Unit & Value \\
\hline $\mathrm{Q}$ & Pos noises (X/Y/Z) & $\mathrm{m}$ & 0.5 \\
& Accel noises (X/Y/Z) & $\mathrm{m} / \mathrm{s}^{2} / \sqrt{\mathrm{Hz}}$ & 0.1 \\
& Gyro noises (R/P/Y) & $\mathrm{rad} / \mathrm{s} / \sqrt{\mathrm{Hz}}$ & 0.001 \\
$\mathrm{R}$ & Range noise & $\mathrm{m}$ & $\geq 20$ \\
& Bearing/Elevation noises & $\circ$ & $0.1604,0.1206$ \\
\hline
\end{tabular}

SLAM update. The display thread fetches the SLAM results and sends them to the ground station and mission computer. The interrupt handler wakes up with the arrival of a synchronisation pulse from the FCC with the corresponding timing pulse from the network.

Table 1 presents the filter $\mathrm{Q} / \mathrm{R}$ tuning parameters used for real-time operation. The range uncertainty was actually estimated within the vision processing node, based on the known feature sizes. The nominal accuracy of the range, during level flight with $100 \mathrm{~m}$ of altitude above ground, was greater than $20 \mathrm{~m}$. Filter initialisation was performed on the fly, utilising information received from GNSS/Inertial system.

During real-time operation, the IMU and vision data are processed in real-time or near real-time, using processing methods depending on the latency in the SLAM filter update. That is, SLAM prediction and update are performed in real-time 
Table 2

SLAM initial parameters and associated uncertainties $(1 \sigma)$ transferred from the on-board FCC system (UTM coordinates are used)

\begin{tabular}{|c|c|c|c|c|c|c|}
\hline State & $\mathrm{X}$ & $\mathrm{Y}$ & $\mathrm{Z}$ & $\mathrm{X}(1 \sigma)$ & $\mathrm{Y}(1 \sigma)$ & $\mathrm{Z}(1 \sigma)$ \\
\hline Position (m) & 6167423.13 & 230258.39 & -769.60 & 2.0871 & 2.1176 & 3.7197 \\
\hline Velocity (m/s) & 31.16 & 15.50 & -3.93 & 1.2488 & 1.1590 & 1.3404 \\
\hline Attitude $\left({ }^{\circ}\right)$ & -11.51 & 12.15 & 6.52 & 1.2194 & 1.3126 & 1.4576 \\
\hline
\end{tabular}

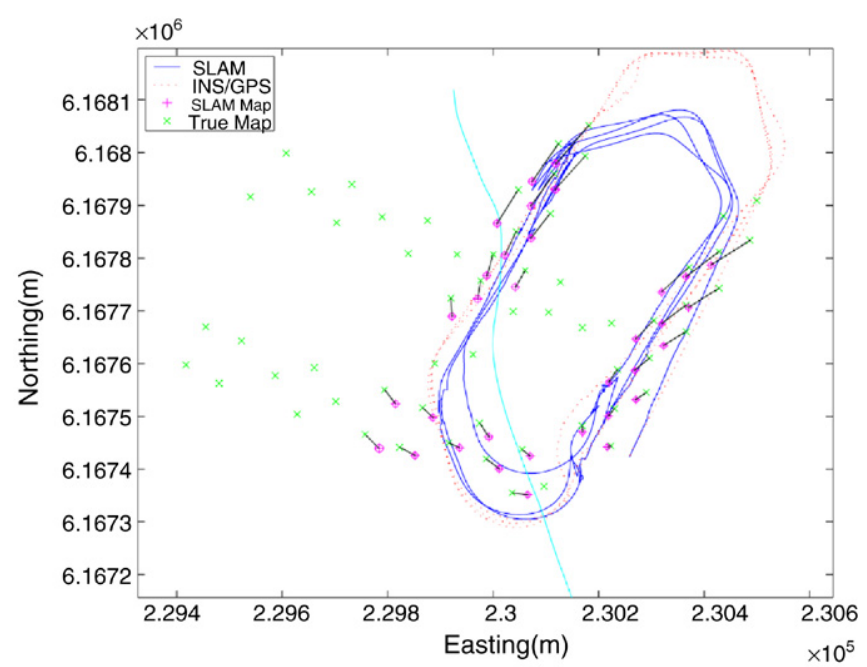

Fig. 4. The vehicle and feature positions estimated from the real-time SLAM system. The GNSS/Inertial position and surveyed feature positions are plotted for comparison, showing the correspondences with the ground truths. $1 \sigma$ ellipsoidal bounds were used for the feature uncertainty.

with a low number of features, but if the latency of the filter update becomes significant, then accumulated inertial/vision data are processed as a block. Another concern for realtime SLAM was about its capacity for reliable loop-closure. Although the ground features were installed with enough separation $(>50 \mathrm{~m})$ to relieve the on-line data association problem, it was observed that loop closure was still unreliable, due to the large drift errors in the INS solution. Hence the data association software was modified to exploit the on-board navigation solution for loop-closure.

\section{Real-time results}

Tests were performed at Marulan, the University of Sydney's flight test facility. A typical flight test time was around 30 min and the nominal flight height was $100 \mathrm{~m}$ above ground. The maximum ground speed was approximately $40 \mathrm{~m} / \mathrm{s}$. In a subsequent flight trial, SLAM was tested as a realtime navigation system. SLAM was activated during flight, when the vision camera had a clear view of features on the ground and when a designated height above ground had been reached. SLAM initialisation required initial position, velocity and attitude information, along with the uncertainty in these values. Table 2 shows these parameters, which were transferred from the onboard GNSS/INS before SLAM was activated. Calibration data for the IMU were also transferred, via the vehicle bus.
The SLAM-estimated vehicle and map positions in real-time are shown in Fig. 4, with the GNSS/Inertial solution also shown for comparison. Both the estimated (' + ' marks) and surveyed (' $X$ ' marks) map positions are also plotted. After take-off, the vehicle underwent autonomous flight in an oval trajectory, and then SLAM was activated from the ground station. The SLAM system incrementally built a map and simultaneously used it to estimate the inertial errors. Fig. 5 presents detailed successive 2D plots of SLAM output during the first round until the vehicle closes the loop, which shows the navigation system incrementally building a map and navigating as the vehicle traverses the flight path. During loop closure, it can be seen that both vehicle and map accuracies improve. Successive reobservation of the features continues to improve the accuracy of the navigation and mapping solution. Fig. 6 also shows the results from subsequent passes. The map uncertainty decreases monotonically towards the lower limit, and it becomes less sensitive to the addition of further information/information gain. This clearly illustrates the success of the implementation and its usefulness for airborne applications.

The difference between the SLAM-estimated vehicle position and the reference GNSS/Inertial solution was partly due to the errors in the initial parameters and the height deviation. The most significant source of error was range calculation in undulating 3D terrain environments. The nonparallel relationship between the plane of the feature and the camera image plane means that the vehicle observes the feature at some angle. Hence, the detected feature dimension is always smaller than its actual value, resulting in a greater-than-actual range estimate. Since the camera detects the new feature in the image plane, this can either push-forward the feature position or push-back the vehicle position/velocity along the longitudinal direction. This effect can be seen more clearly in Fig. 5(d), where the increased vehicle uncertainty, following its first cornering without any observed features, caused a noticeable backward correction. These errors can be overcome by using a more accurate mission sensor and a proper ranging device.

In Fig. 7, the position, velocity and attitude estimates from SLAM are compared with GNSS/Inertial solutions, and the SLAM estimated $1 \sigma$ covariances are plotted. The large errors in position estimates are due to the the low quality inertial system used. The velocity and attitude results are more promising, and comparable to those of the corresponding GNSS/Inertial solutions, showing the ability of the SLAM filter to stabilise INS. Table 3 presents the standard deviations of the SLAM errors, which are obtained by subtracting the results from the GNSS/Inertial outputs and ground surveyed map positions. By comparing these values with the estimated covariances (plotted in Fig. 7), it can be observed that the SLAM filter maintained 


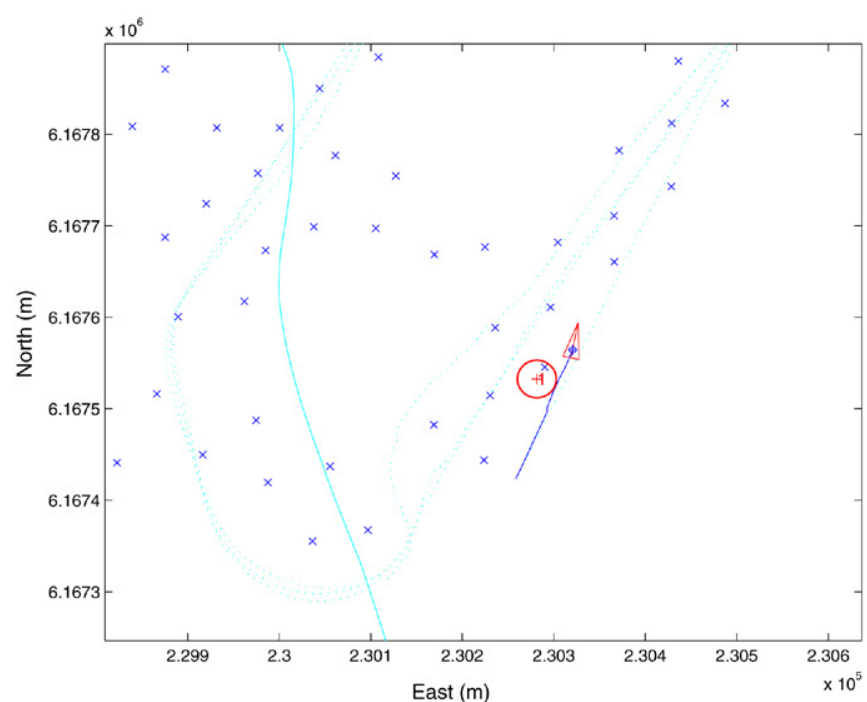

(a)

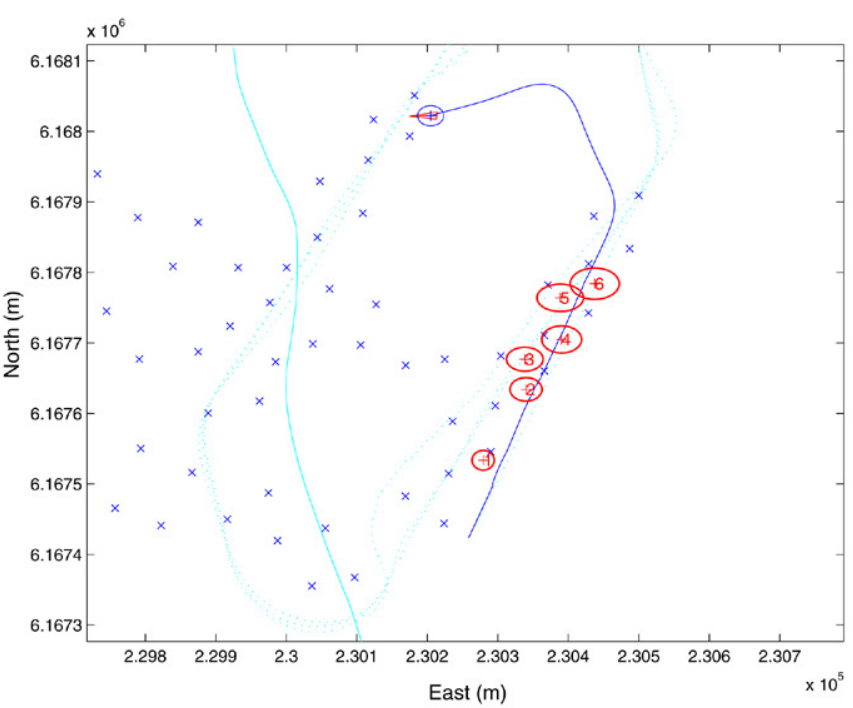

(c)

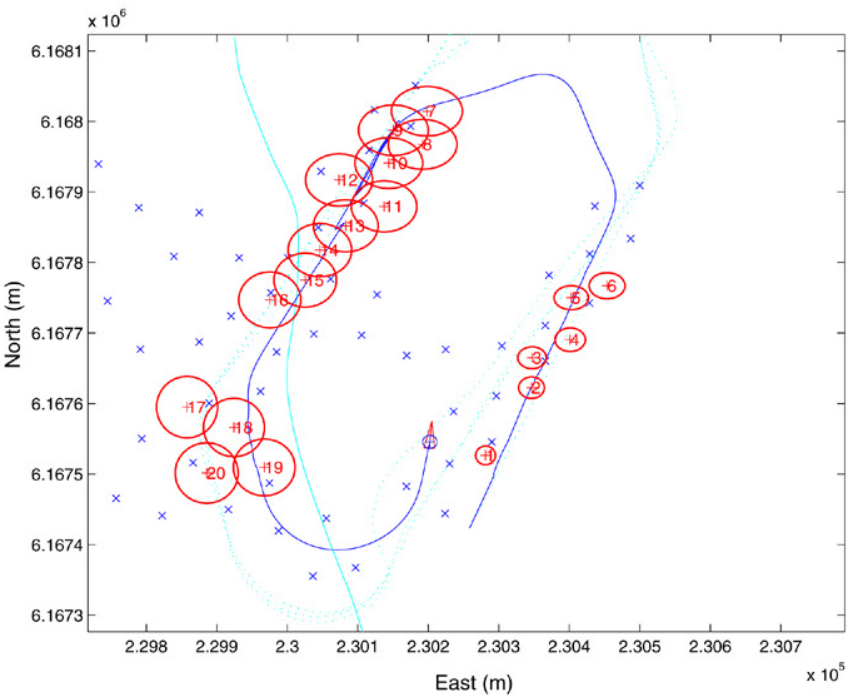

(e)

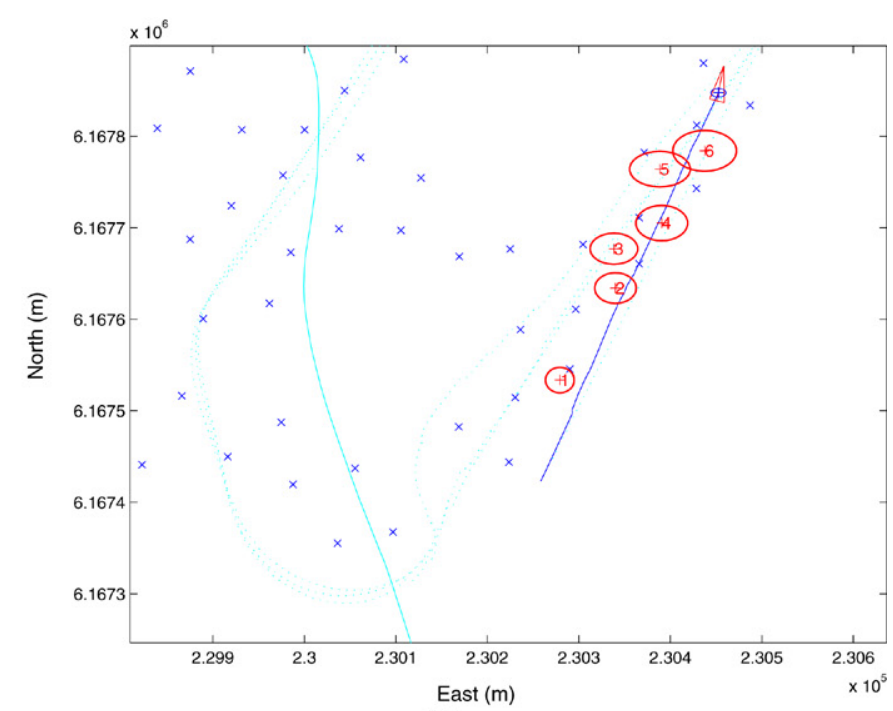

(b)

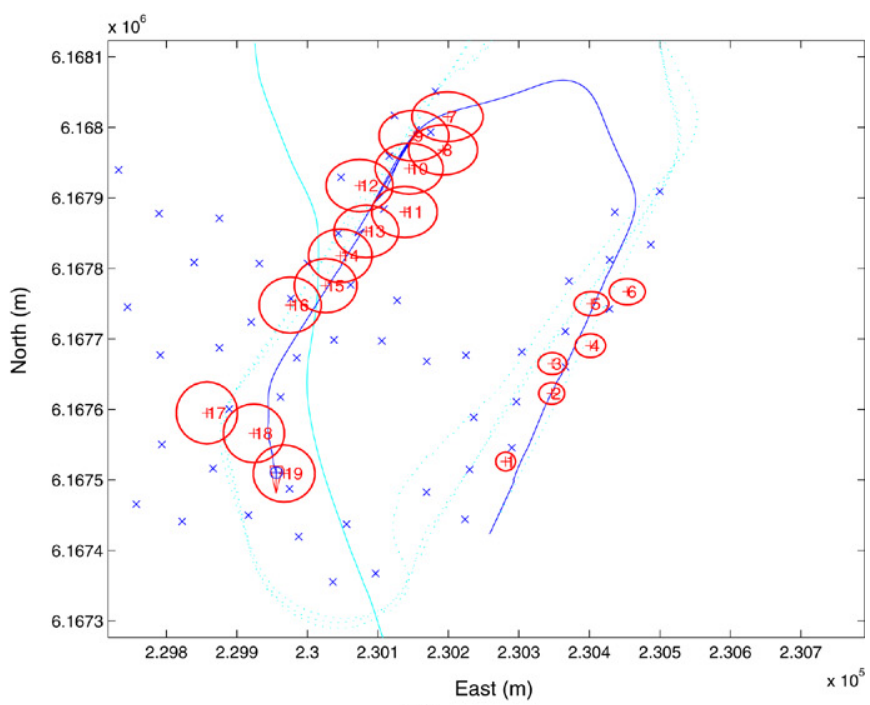

(d)

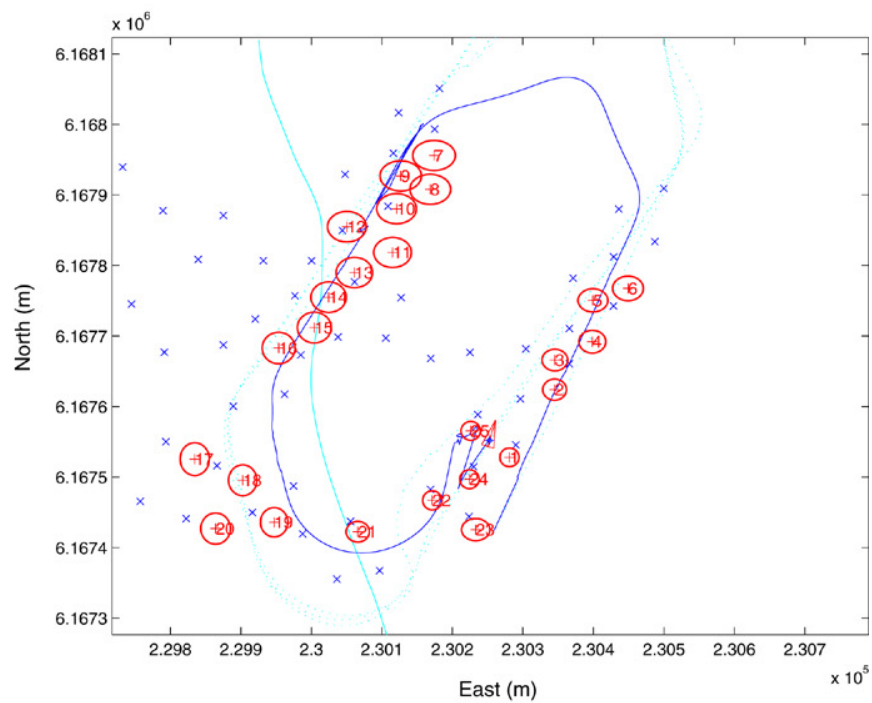

(f)

Fig. 5. The detailed views of real-time SLAM during the first round. (a) The SLAM system was activated during the flight. (b), (c) and (d) present the incremental building up of the map, which is supposed to estimate inertial errors simultaneously ( $5 \sigma$ ellipses were plotted for the feature). (e) shows the vehicle approaching the initial position, and in (f) the loop is closed by observing previous features. Both map and vehicle accuracies can be seen to have improved. 


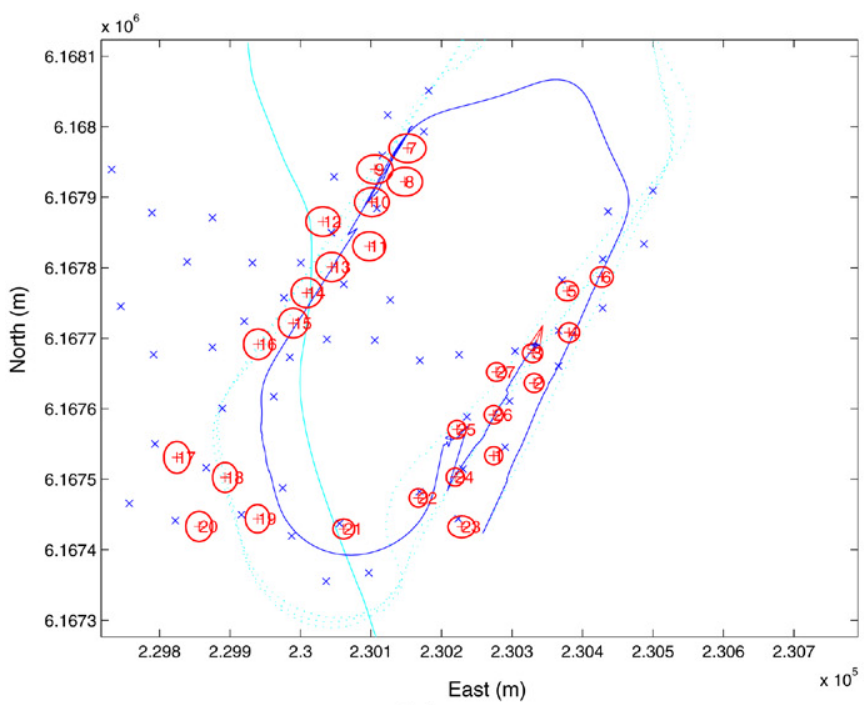

(a)

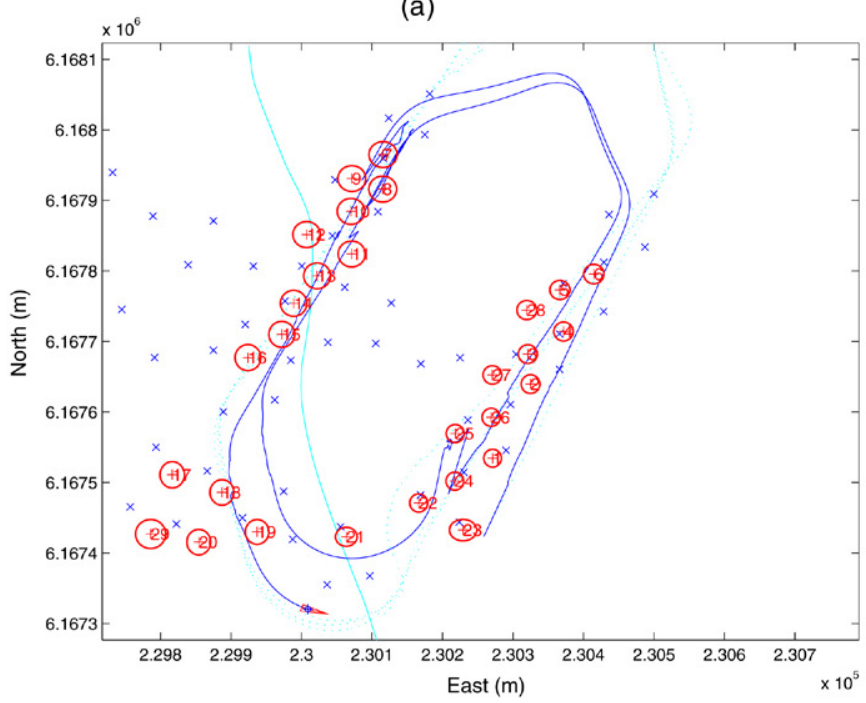

(c)

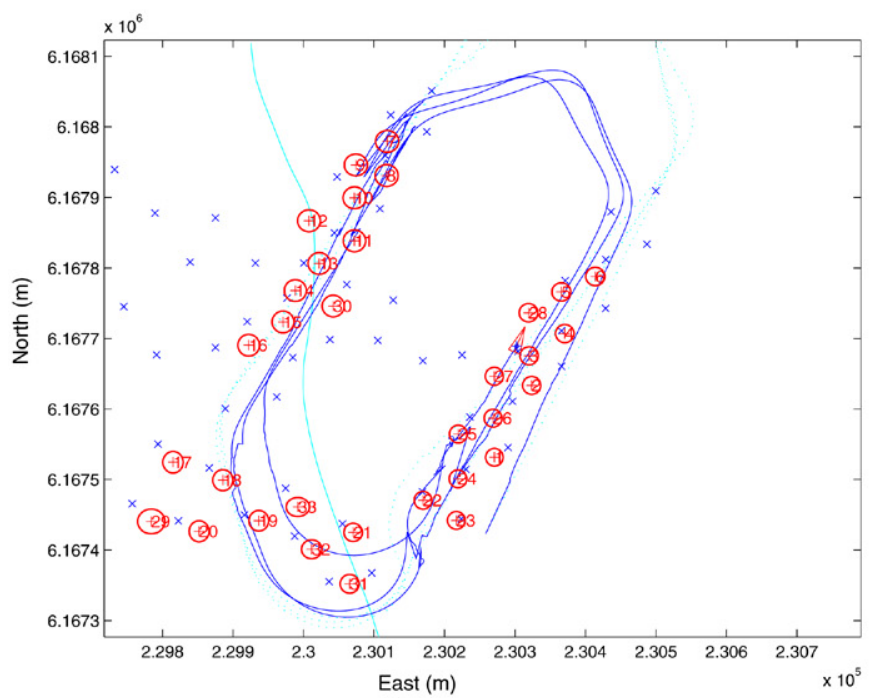

(e)

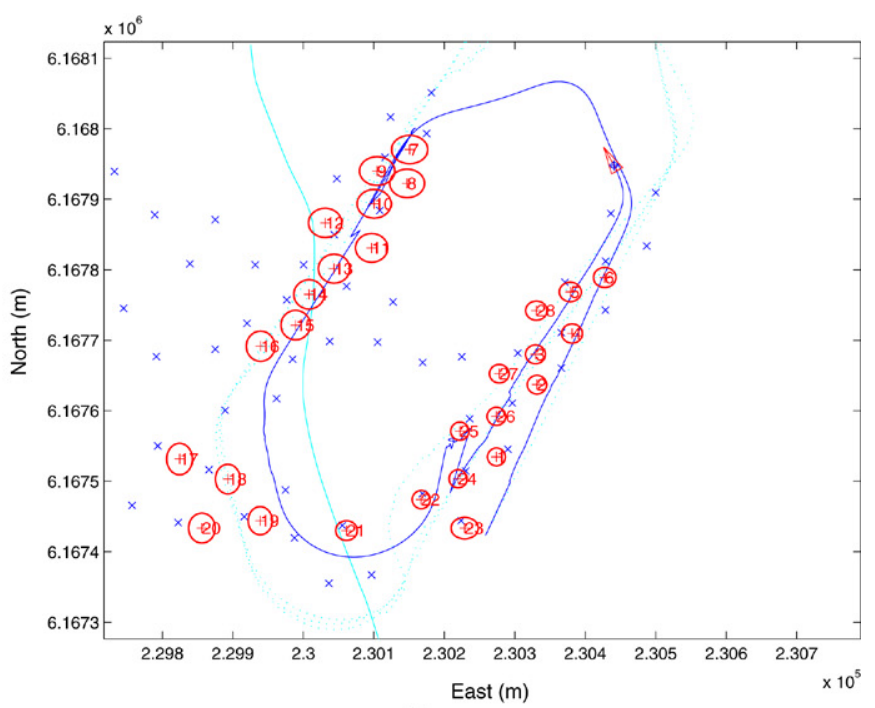

(b)

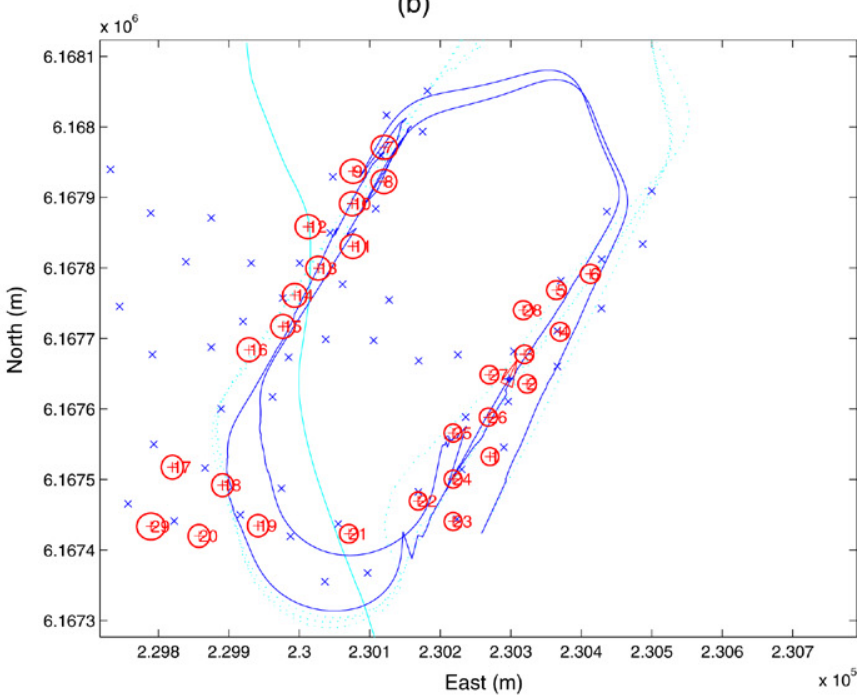

(d)

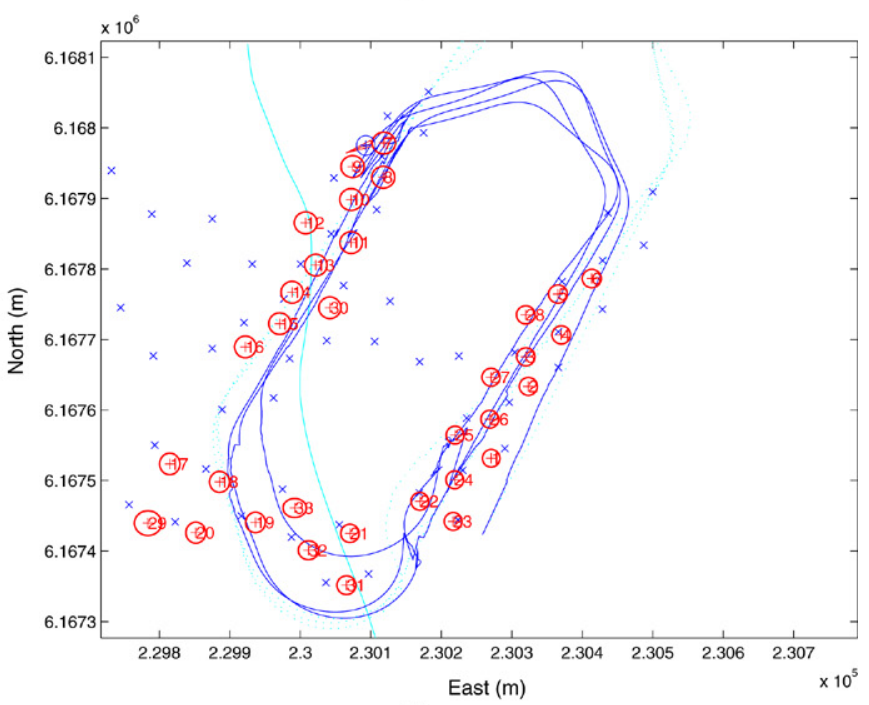

(f)

Fig. 6. Detailed views of real-time SLAM during successive loops. The plots show that the uncertainties of the vehicle and map further decrease monotonically due to successive re-observations and loop closures. 

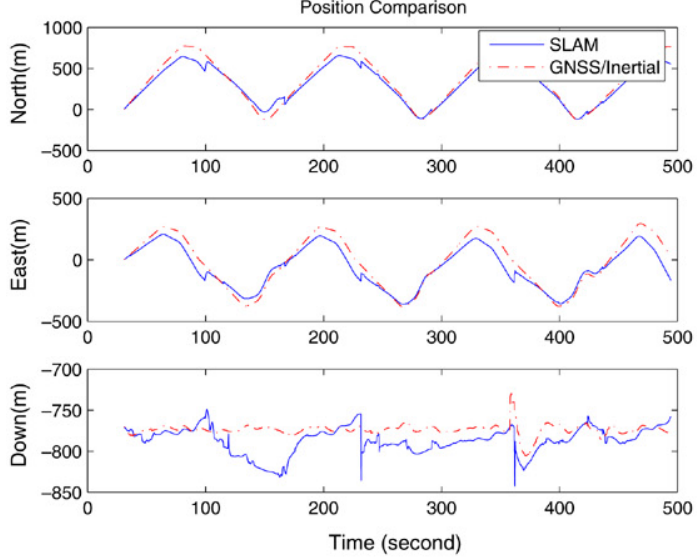

(a)
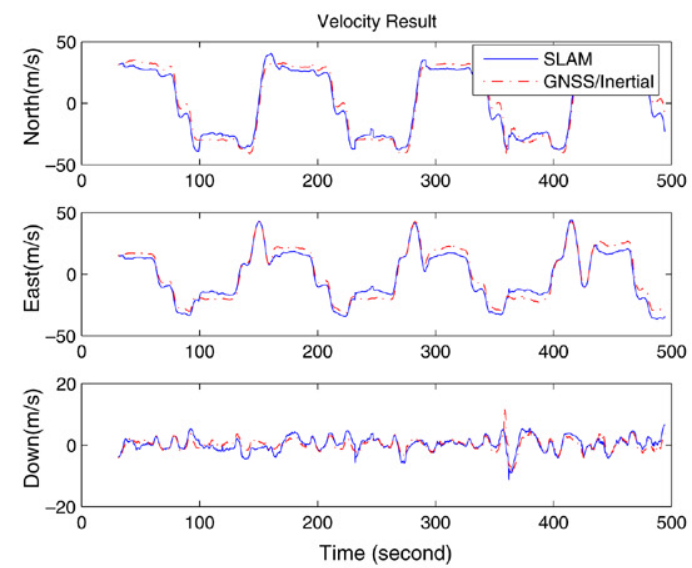

(c)
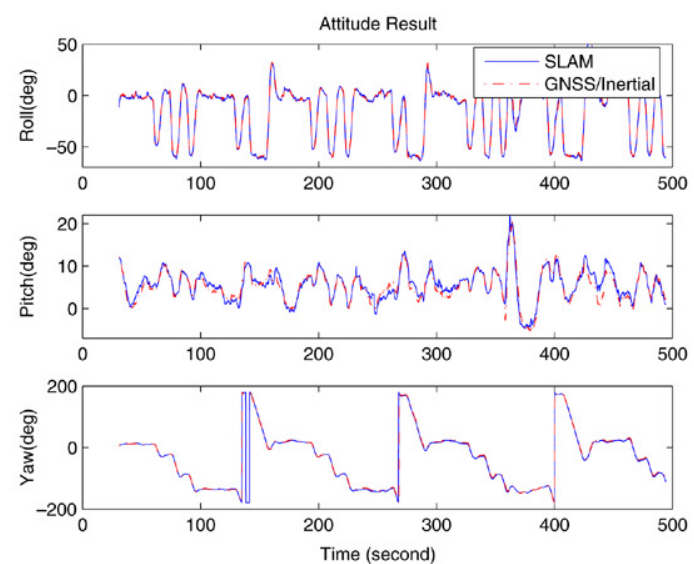

(e)

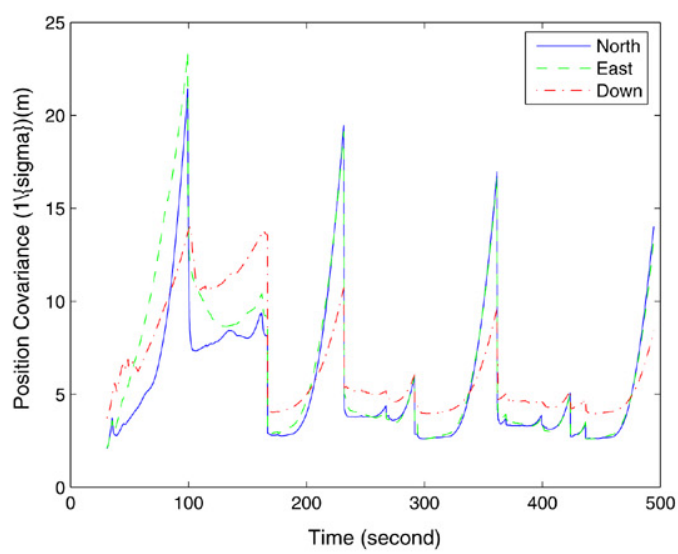

(b)

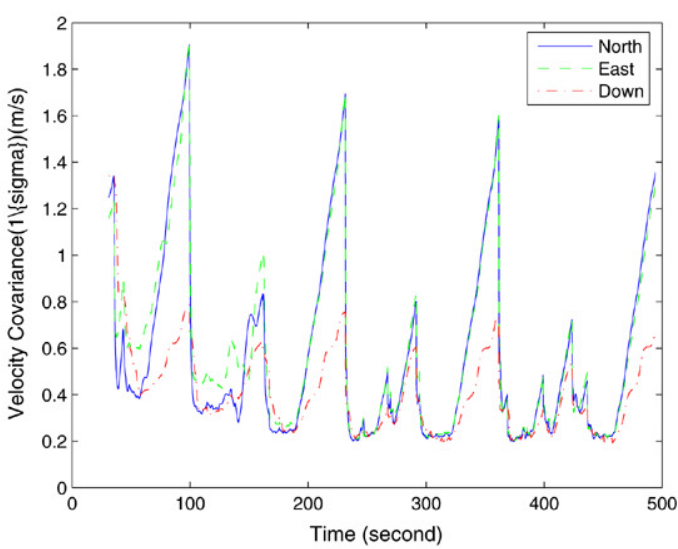

(d)

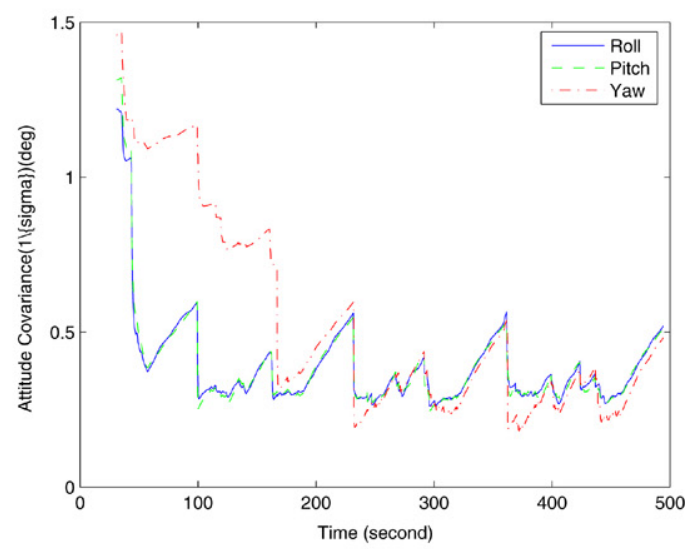

(f)

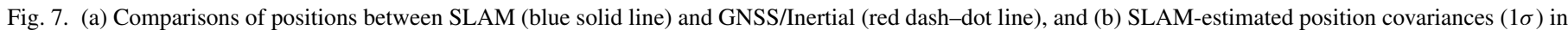

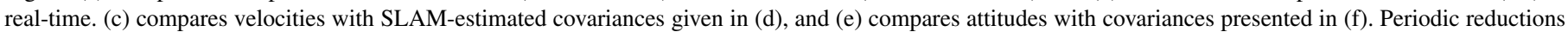

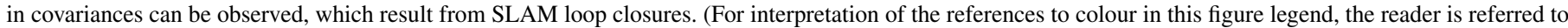
the web version of this article.)

over-confident covariance estimates. This can be improved by performing a more precise error analysis, using the errors arising from the inertial sensors and during initialisation. However, as the process noises increase, the vehicle states, particulary the attitude, become more sensitive to SLAM observation updates. This can be overcome either by partitioning the attitude updates from the velocity/position ones, or by fusing them with an ex- tra aiding source, such as an air-data system, to improve INS performance between vision updates, and to maintain the uncertainty within an acceptable level.

There are still a number of theoretical, technical, and practical issues that need to be resolved, including SLAM consistency, data synchronisation between vision and INS, real-time implementation of the indirect filter, natural feature 
Table 3

Standard deviations of SLAM errors computed by subtracting SLAM estimates from GNSS/Inertial solutions

\begin{tabular}{lrrr}
\hline Errors & $\mathrm{X}(1 \sigma)$ & $\mathrm{Y}(1 \sigma)$ & $\mathrm{Z}(1 \sigma)$ \\
\hline Position $(\mathrm{m})$ & 64.44 & 60.93 & 15.59 \\
Velocity $(\mathrm{m} / \mathrm{s})$ & 5.01 & 4.46 & 1.49 \\
Attitude $\left(^{\circ}\right)$ & 1.35 & 1.15 & 1.89 \\
Map $(\mathrm{m})$ & 19.45 & 31.98 & 30.29 \\
\hline
\end{tabular}

detection and representation, and the incorporation of sub-map techniques for large scale deployment.

\section{Conclusions}

This paper presented the results of a real-time implementation of airborne SLAM on a UAV platform by fusing inertial data with information from a vision sensor. Although airborne SLAM is still in its infancy, with many exciting areas for future research, the results presented here have clearly illustrated its capability as a reliable and accurate airborne navigation and mapping system. SLAM consistency and robustness needs to be further investigated. It was observed that any inconsistent or overconfident attitude updates would severely affect any SLAM solution. This problem is currently being addressed by decoupling attitude updates from position/velocity updates, or by fusing additional velocity observations, such as air velocity observations, to make airborne SLAM less susceptible and to enhance its robustness.

\section{References}

[1] B. Parkinson, Origins, evolution, and future of satellite navigation, Journal of Guidance, Control, and Dynamics 20 (1) (1997) 11-25.

[2] J. Kim, S. Sukkarieh, S. Wishart, Real-time navigation, guidance and control of a UAV using low-cost sensors, in: International Conference of Field and Service Robotics, Yamanashi, Japan, 2003, pp. 95-100.

[3] R. Smith, M. Self, P. Cheeseman, Estimating Uncertain Spatial Relationships in Robotics, vol. 2, Elsevier Science, 1988.

[4] J. Guivant, E. Nebot, Optimization of the simultaneous localisation and map building algorithm for real-time implementation, IEEE Transactions on Robotics and Automation 17 (3) (2001) 242-257.

[5] S. Williams, M. Dissanayake, H. Durrant-Whyte, An Efficient approach to the simultaneous localisation and mapping problem, in: IEEE
International Conference on Robotics and Automation, vol. 1, 2002, pp. 406-411.

[6] C. Estrada, J. Neira, J. Tardos, Hierarchical SLAM: Real-time accurate mapping of large environments, IEEE Transactions on Robotics 21 (4) (2005) 588-596.

[7] S. Thrun, Y. Liu, D. Koller, A. Ng, Z. Ghahramani, H. Durrant-Whyte, Simultaneous localization and mapping with sparse extended information filters, International Journal of Robotics Research 23 (2004) 693-716.

[8] A. Nuchter, H. Surmann, K. Lingemann, J. Hertzberg, S. Thurn, 6D SLAM with an application in autonomous mine mapping, IEEE Transactions on Robotics and Automation (2004) 1998-2003.

[9] I. Jung, S. Lacroix, High resolution terrain mapping using low altitude aerial stereo imagery, in: The Ninth IEEE International Conference on Computer Vision, 2003.

[10] J. Langelaan, S. Rock, Passive GPS-free navigation for small UAVs, in: 2005 IEEE Conference on Aerospace, 2005, pp. 1-9.

[11] J. Kim, S. Sukkarieh, Autonomous airborne navigation in unknown terrain environments, IEEE Transactions on Aerospace and Electronic Systems 40 (3) (2004) 1031-1045.

[12] P. Savage, Strapdown Analytic: Part 1, 2, Strapdown Associates, Inc., 2000.

[13] D. Titterton, Strapdown Inertial Navigation Technology, Peter Peregrinus, Ltd., 1997.

[14] J. Kim, S. Sukkarieh, Recasting SLAM — Towards improving efficiency and platform independency, in: Robotics Research: The 11th International Symposium, Springer-Verlag, 2005.

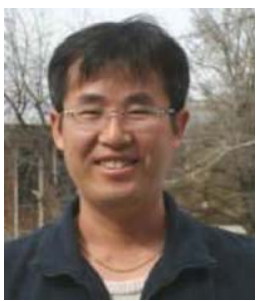

Dr. Jonghyuk Kim is currently a Lecturer at the Australian National University in the Faculty of Engineering and Information Technology. He obtained his Ph.D. degree at the University of Sydney in 2004 researching in the area of autonomous airborne navigation. He was a postdoctoral fellow at the Centre for Autonomous Systems in Sydney, developing airborne SLAM (Simultaneous Localisation and Mapping) and integrated navigation systems. His current research areas include airborne SLAM, cooperative navigation, and decentralised aerial robotics.

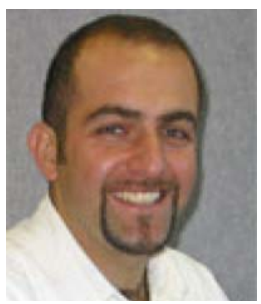

Dr. Salah Sukkarieh is a Senior Lecturer at the University of Sydney in the School of Aerospace, Mechanical and Mechatronic Engineering and is the Programme Leader for Aerospace at the Australian Centre for Field Robotics in the ARC Centre of Excellence for Autonomous Systems. He obtained his Ph.D. in 2000 at the University of Sydney researching in the area of inertial navigation. His current research areas include SLAM for airborne and ground platforms, cooperative control, and Systems of Systems Design. 